\title{
Tigecycline and intravenous fosfomycin zone breakpoints equivalent to the EUCAST MIC criteria for Enterobacteriaceae
}

\author{
Fernando Pasteran, Celeste Lucero, Melina Rapoport, Leonor Guerriero, Irene Barreiro, \\ Ezequiel Albornoz, Omar Veliz, Alejandra Corso
}

\begin{abstract}
Servicio Antimicrobianos, Departamento Bacteriología, Instituto Nacional de Enfermedades Infecciosas (INEI)ANLIS "Dr. Carlos G. Malbrán", Ministerio de Salud y Ambiente, Ciudad Autónoma de Buenos Aires, Argentina
\end{abstract}

\begin{abstract}
Introduction: Tigecycline and intravenous (i.v.) fosfomycin could be alternative therapeutic options for the treatment of carbapenemasepossessing Enterobacteriaceae bacterial infections. However, routine laboratories are forced to test these drugs using minimum inhibitory concentration (MIC) methods as zone breakpoints are not available for the disc diffusion technique.

Methodology: Clinical and Laboratory Standards Institute methods for agar dilution and disc diffusion were compared to determine tentative zone breakpoints that best correlate to tigecycline and i.v. fosfomycin MIC breakpoints defined by the European Committee on Antimicrobial Susceptibility Testing. A total of 195 Enterobacteriaceae with defined mechanisms of resistance were tested in duplicate assays. Half of the strains were characterized as carbapenemase producers (KPC-2, OXA-48, OXA-163, VIM-1, VIM-2, IMP-8, NDM-1).

Results: Corresponding zone diameters of susceptible $\geq 15 \mathrm{~mm}$, resistant $\leq 12 \mathrm{~mm}$ and susceptible $\geq 17 \mathrm{~mm}$, resistant $\leq 15 \mathrm{~mm}$ for the $50 \mu \mathrm{g}$ fosfomycin plus $50 \mu \mathrm{g}$ glucose-6-phosphate and $200 \mu \mathrm{g}$ fosfomycin plus $50 \mu \mathrm{g}$ glucose-6-phosphate discs, respectively, allowed categorization of the strains with an acceptable level of error $(<10 \%$ minor errors, $<1.5 \%$ major errors, $<1 \%$ very major errors and categorical agreement $>90 \%$ ). For the $15 \mu \mathrm{g}$ tigecycline disc, the best performance was achieved with the corresponding zone diameters of susceptible $\geq 21 \mathrm{~mm}$ and resistant $\leq 16 \mathrm{~mm}$, which eliminated the very major and major errors but not the minor errors $(34.4 \%)$.

Conclusions: Based on these results, tigecycline and fosfomycin can be included in the routine panel of antibiotics for susceptibility testing by disc diffusion to provide fast and reliable information for the selection of treatment alternatives, especially for strains with extreme resistance, as carbapenemase producers.
\end{abstract}

Key words: tigecylcine; fosfomycin; disc diffusion; KPC; carbapenemase

J Infect Dev Ctries 2012; 6(5):452-456.

(Received 04 August 2011 - Accepted 07 November 2011)

Copyright (C) 2012 Pasteran et al. This is an open-access article distributed under the Creative Commons Attribution License, which permits unrestricted use, distribution, and reproduction in any medium, provided the original work is properly cited

\section{Introduction}

The emergence of carbapenemase-possessing Enterobacteriaceae has resulted in extremely limited therapeutic options. Tigecycline, a broad-spectrum glycylcycline, may be a beneficial and safe adjunctive treatment in the management of lifethreatening infections caused by carbapenemresistant Klebsiella pneumoniae [1]. Clinicians have also reconsidered the potential value of old antibiotic compounds as intravenous (i.v.) fosfomycin (disodium salt) in the treatment of multidrug resistant Gram-negative bacterial infections [2].

Updated susceptibility data are vital in guiding the selection of treatment with these agents; however, interpretation of these data depends on the availability of reliable breakpoints. The European Committee on Antimicrobial Susceptibility Testing/European Medicines Agency
(EUCAST/EMA) has defined both tigecycline and fosfomycin i.v. MIC breakpoints for Enterobacteriaceae as follows: tigecycline; susceptible $\leq 1.0 \mathrm{mg} / \mathrm{L}$ and resistant $\geq 4.0 \mathrm{mg} / \mathrm{L}$; fosfomycin i.v.: susceptible $\leq 32 \mathrm{mg} / \mathrm{L}$ and resistant $\geq 64 \mathrm{mg} / \mathrm{L}$ [3], On the other hand, the Clinical and Laboratory Standards Institute (CLSI) has yet to set values; breakpoints are available only for fosfomycin trometamol, an oral formulation intended for Escherichia coli urinary tract infection treatment [4].

Considering that MIC is a time-consuming method, that several automated systems have not yet included tigecycline and fosfomycin, and that the Etest has shown conflicting results $[5,6]$, disc diffusion seems to be the most practical alternative in routine labs to evaluate susceptibility to these agents. EUCAST has defined tigecycline zone breakpoints only for $E$. coli (susceptible $\geq 18 \mathrm{~mm}$ and resistant $\leq$ 
Table 1. Comparison of categorical agreement and errors using different disc zone breakpoints

\begin{tabular}{|c|c|c|c|c|c|c|}
\hline Disc & Breakpoint & Source & $\mathrm{CA}(\%)$ & VME(\%) & MaE(\%) & MiE(\%) \\
\hline Tigecycline $15 \mu \mathrm{g}$ & $\mathrm{S} \geq 18 \mathrm{R} \leq 14$ & EUCAST & 43.1 & 4.6 & 0 & 52.3 \\
\hline Tigecycline $15 \mu \mathrm{g}$ & $\mathrm{S} \geq 21 \mathrm{R} \leq 17$ & Hope et al. [8] & 65.6 & 0 & 2.1 & 32.3 \\
\hline $\begin{array}{c}\text { Tigecycline } \\
15 \mu \mathrm{g}\end{array}$ & $S \geq 21 \quad R \leq 16$ & This work & 65.6 & $\mathbf{0}$ & $\mathbf{0}$ & 34.4 \\
\hline $\begin{array}{c}\text { Fosfomycin } \\
200 / 50 \mu g^{a}\end{array}$ & $\mathrm{~S} \geq 14 \mathrm{R} \leq 10^{\mathrm{b}}$ & Lu et al. [9] & 95.3 & 4.1 & 0.6 & 0 \\
\hline $\begin{array}{c}\text { Fosfomycin } \\
200 / 50 \mu \mathrm{g}\end{array}$ & $\mathrm{S} \geq 14 \mathrm{R} \leq 13^{\mathrm{b}}$ & Lu et al. [9] & 94.7 & 4.7 & 0.6 & 0 \\
\hline $\begin{array}{c}\text { Fosfomycin } \\
200 / 50 \mu \mathrm{g}\end{array}$ & $\mathrm{S} \geq 16 \mathrm{R} \leq 13^{\mathrm{c}}$ & Lu et al. [9] & 96.2 & 2.8 & 1 & 0 \\
\hline $\begin{array}{c}\text { Fosfomycin } \\
200 / 50 \mu \mathrm{g} \\
\end{array}$ & $S \geq 17 \quad R \leq 15^{d}$ & This work & 92.3 & $\mathbf{0}$ & 0.6 & 7.2 \\
\hline $\begin{array}{c}\text { Fosfomycin } \\
50 / 50 \mu \mathrm{g}^{\mathrm{e}}\end{array}$ & $S \geq 15 \quad R \leq 12^{d}$ & This work & 90.6 & $\mathbf{0}$ & 1.1 & 8.3 \\
\hline
\end{tabular}

CA, categorical agreement; VME, very major errors; MaE, major errors; MiE, minor errors. S, susceptible; R, resistant.

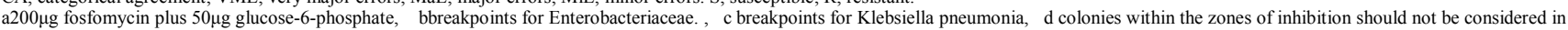
the zone diameter determination

$14 \mathrm{~mm}$ ) [3]. Unfortunately, Klebsiella pneumoniae, which is more likely to develop extreme drug resistance (XDR, i.e., resistance to all but two or fewer antimicrobial categories) than E. coli [7], is not included in these standards. A recent publication showed that, for Enterobacteriaceae, tigecycline zone breakpoints of susceptible $\geq 21 \mathrm{~mm}$ and resistant $\leq 17 \mathrm{~mm}$ produced the best agreement with the EUCAST MIC categorization [8]. Fosfomycin i.v. faces a more complex scenario because discs containing different loads ( $200 \mu \mathrm{g}$ of fosfomycin plus $50 \mu \mathrm{g}$ glucose-6-phosphate and $50 \mu \mathrm{g}$ fosfomycin plus $50 \mu \mathrm{g}$ glucose-6-phosphate) are available (glucose-6-phosphate is required for induction of the transport system necessary for entry of fosfomycin into bacterial cells). Fosfomycin zone breakpoints corresponding to EUCAST MIC were recently proposed by $\mathrm{Lu}$ et al. for the $200 \mu \mathrm{g}$ of fosfomycin disc (susceptible $\geq 14 \mathrm{~mm}$ and resistant $\leq 10 \mathrm{~mm}$ or susceptible $\geq 14 \mathrm{~mm}$ and resistant $\leq 13 \mathrm{~mm}$ for species of Enterobacteriaceae) [9].Thus there is an urgent international need to have tigecycline and fosfomycin cutoffs validated in more than one center, by using strains belonging to a presumably different bacterial population, especially XDR isolates, and establishing zone breakpoint corresponding to EUCAST MIC cutoff values for the $50 \mu \mathrm{g}$ fosfomycin disc.

The present study was designed to i) optimize the recently proposed tigecycline and fosfomycin zone breakpoints by challenging them with a panel of XDR strains, and ii) determine fosfomycin i.v zone breakpoints by the CLSI diffusion method for the 50 $\mu \mathrm{g}$ fosfomycin disc that best correlates with the EUCAST MIC breakpoints.

\section{Methodology}

A panel of 195 Enterobacteriaceae (158 K. pneumoniae, 24 Enterobacter spp., 6 Serratia marcescens, 3 Citrobacter freundii, 2 Klebsiella oxytoca, 1 Escherichia coli, and 1 Providencia rettgeri) were tested for tigecycline. A subset of 181 isolates was further evaluated for fosfomycin. The strains were submitted to the National Reference Laboratory because they either had an XDR phenotype $(50 \%$ of the isolates) or required a tigecycline/fosfomycin MIC confirmation. The resistant mechanisms were characterized by PCR and DNA sequencing and included (n): KPC (70), CTXM-2 plus porin loss (21), OXA-163 (3), IMP-8 (3), VIM-2 (2), VIM-1 (1), NDM-1 (1), OXA-48-like (1), ESBLs (CTXM-2, PER-2, SHV-2, SHV-5, SHV-18) (76), narrow-spectrum beta-lactamases (6), and wild type isolates (including ATCC quality control strains) (12). The isolates were from clinical sources and they were single isolates from each patient. The strains were recovered from 65 hospitals located in 25 cities from 17 Provinces (2007-2010 period).

The MIC of fosfomycin (Sigma) and tigecylcine (Pfizer) were determined by the agar dilution method using Mueller-Hinton medium (Difco, BectonDickinson) according to CLSI recommendations [10]. For fosfomycin, the agar was supplemented with $25 \mathrm{mg} / \mathrm{L}$ of glucose-6-phosphate (Sigma) [4]. Simultaneously, $15 \mu \mathrm{g}$ tigecycline (Oxoid), $200 \mu \mathrm{g}$ fosfomycin plus $50 \mu \mathrm{g}$ glucose-6-phosphate (BectonDickinson) and $50 \mu \mathrm{g}$ fosfomycin plus $50 \mu \mathrm{g}$ glucose- 
Figure 1. Scattergrams showing antimicrobial susceptibility results obtained with disc diffusion (in mm) and agar dilution

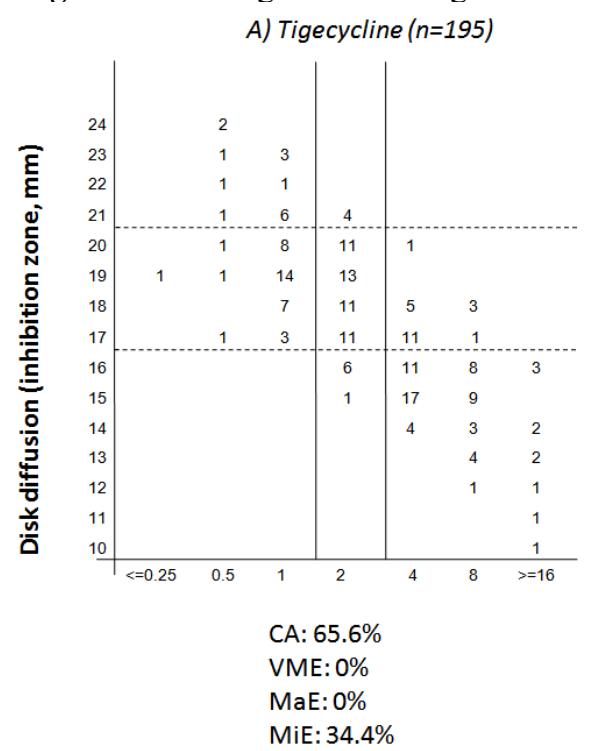

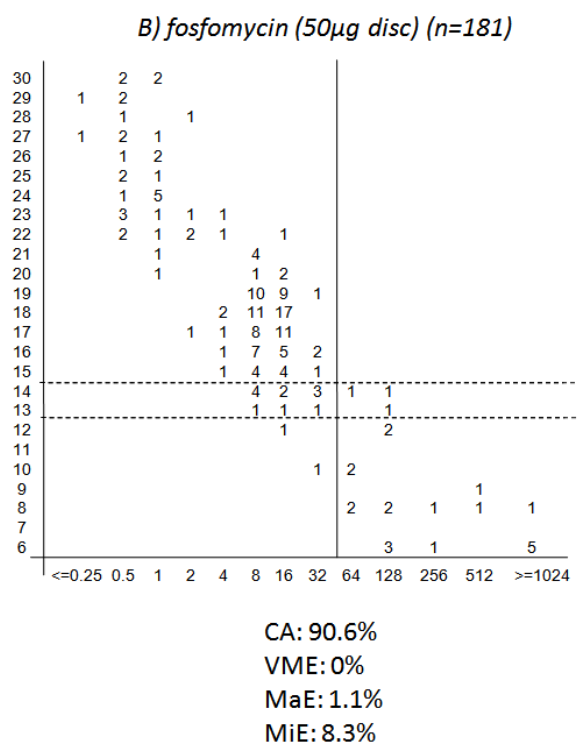

Agar dilution (MIC, mg/L) 6-phosphate (Oxoid) discs were tested following the method outlined by CLSI using Mueller-Hinton medium (Difco, Becton-Dickinson) [11]. Strains were tested in duplicate assays and the average result was recorded.

\section{Results and discussion}

The results obtained by challenging the panel of strains with different breakpoints are shown in Table 1. By error minimization analysis we did not find a tigecycline zone breakpoint that could reduce errors to acceptable levels $(<10 \%$ minor errors $-\mathrm{MiE}-,<$ $1.5 \%$ major errors-MaE-, $<1 \%$ very major errorsVME- and categorical agreement-CA- > 90\%). Tigecycline EUCAST zone breakpoints for E. coli led to unacceptable levels of errors. When we challenged the panel of strains included in this study with the zone breakpoints recommended by Hope et al. [8], we observed a larger amount of $\mathrm{MaE}(2.1 \%)$ and $\mathrm{MiE}(32.3 \%)$ with respect to those previously reported by this author (CA $75.5 \%$, VME $0.6 \%$, MaE $0.6 \%$, MiE $23.3 \%$ ). These observed differences could be due to the different brands of Mueller-Hinton agar used. Even so, this zone breakpoint was one of the two cutoff values that displayed the best performance. We found an alternative zone breakpoint of susceptible $\geq 21 \mathrm{~mm}$ and resistant $\leq 16$ $\mathrm{mm}$ that showed a similar number of total errors but eliminated the MaE (Figure 1 and Table 1). As the elimination of a potential agent for treatment may be critical for XDR strains, the reduction of MaE errors was prioritized in this study; thus this breakpoint was further selected. Clinical labs using disc diffusion on a daily basis could properly categorize true tigecycline susceptible and resistant strains by using a zone breakpoint of susceptible $\geq 21 \mathrm{~mm}$ and resistant $\leq 16 \mathrm{~mm}$ (VME and MaE 0\%) (Mueller-Hinton agar from Difco, BD, New Jersey, US). Strains with halos between 17-20 mm could fall in any of the three MIC categories, although most of the isolates had a trend toward susceptibility by the reference (MIC) method, as has been also observed in other study using the BSAC method [12]. We observed in this series that $40 \%$ of strains with intermediate halos showed susceptibility by MIC, and only $7 \%$ resulted true resistant. Thus strains with halos of 17-20 mm must be confirmed by MIC before removing tigecycline prematurely as a therapeutic option.

As panels can introduce bias, we analyzed the impact of the selected tigecycline zone breakpoints in a clinical scenario by examining the halos distribution of nosocomial Enterobacteriaceae obtained by 92 Hospitals (WHONET-Argentina Network; $n=5605$, year 2009-2010). In this setting, the actual number of isolates that will require a definition by MIC (halos 17-20 mm) was 34\%, not very different from that observed for the panel, while the remaining $66 \%$ will be properly classified as true 
susceptible (halos $\geq 21 \mathrm{~mm}, 63 \%$ of the strains) or true resistant (halos $\leq 16 \mathrm{~mm}, 3 \%$ ).

For the $200 \mu \mathrm{g}$ fosfomycin disc, when we challenged the panel of strains included in this study with the zone breakpoints recommended by Lu et al. [8], we observed a similar amount of VME (4.1\%) to those previously reported by this author (VME 3.7\%) (Table 1). For this disc load, we found an alternative zone breakpoint of susceptible $\geq 17 \mathrm{~mm}$ and resistant $\leq 15 \mathrm{~mm}$ that reduced VME to an acceptable level $(0 \%)$ (Figure 1 and Table 1). On the other hand, fosfomycin $50 \mu \mathrm{g}$ disc zone breakpoints of susceptible $\geq 15 \mathrm{~mm}$ and resistant $\leq 12 \mathrm{~mm}$ produced the best agreement with EUCAST MIC (i.v. formulation) categorization (Figure 1 and Table 1). Proper interpretation of the fosfomycin breakpoints proposed here requires that colonies within the zones of inhibition should not be considered in the zone diameter determination. Based on our results, the disc diffusion method, regardless of the disc load $(50 \mu \mathrm{g}$ or $200 \mu \mathrm{g}$ ), resulted highly reliable for MIC categorization with EUCAST breakpoints for i.v. use. In summary, for fosfomycin i.v., targeting optimal therapy can be done with the CLSI disc method using the zone breakpoints proposed here with no need to confirm target isolates using an MIC method.

This paper presents some limitations that deserve mention: i) a high proportion of Klebsiella isolates were included in this study. As these bacteria very frequently tend to become XDR, it is likely that the current number of bacterial species that require a tigecycline and fosfomycin test in the clinical laboratory will be quite similar; ii) the cutoff values were obtained with Mueller-Hinton agar from Difco. It was recently reported that different Mueller-Hinton agars could affect tigecycline categorization among Enterobacteriaceae [13]. Although limited by the low number of strains tested, Difco media showed a trend toward larger inhibition zones in the report by Torrico et al. [13]. Thus users of Mueller-Hinton agar other than Difco can report with high confidence those strains categorized as susceptible with the zone breakpoints proposed in this study.

Based on the results of this work, tigecycline and fosfomycin can be included in the routine panel of antibiotics for susceptibility testing by disc diffusion to provide fast and reliable information for the selection of treatment alternatives for XDR strains.

\section{Acknowledgment}

Part of this work was presented at the 21st European

Congress of Clinical Microbiology and Infectious Diseases and the 27th International Congress of Chemotherapy, Milan, Italy, May 7-10, 2011.

We are indebted to Dr. Roberto Melano for providing VIM-1 and NDM-1 reference strains.

Funding: This work was supported by the regular federal budget of the Ministry of Health of Argentina.

\section{References}

1. Hirsch EB and Tam VH (2010) Detection and treatment options for Klebsiella pneumoniae carbapenemases (KPCs): an emerging cause of multidrug-resistant infection. J Antimicrob Chemother 65: 1119-1125.

2. Michalopoulos A, Virtzili S, Rafailidis P, Chalevelakis G, Damala M, Falagas ME (2010) Intravenous fosfomycin for the treatment of nosocomial infections caused by carbapenem-resistant Klebsiella pneumoniae in critically ill patients: a prospective evaluation. Clin Microbiol Infect 16: 184-186.

3. European Committee on Antimicrobial Susceptibility Testing clinical breakpoints (2011), http://www.eucast.org/clinical_breakpoints/. Accessed 1 August 2011.

4. Clinical and Laboratory Standards Institute (2011) Performance standards for antimicrobial susceptibility testing; informational supplement, 21st ed. CLSI document M100-S21 CLSI, Wayne, PA, USA,

5. Endimiani A, Patel G, Hujer KM, Swaminathan M, Perez F, Rice LB, Jacobs MR, Bonomo RA (2010) In vitro activity of fosfomycin against bla $\mathrm{KPC}_{\mathrm{K}}$-containing Klebsiella pneumoniae isolates, including those nonsusceptible to tigecycline and/or colistin. Antimicrob Agents Chemother 54: 526-529.

6. Cohen Stuart J, Mouton JW, Diederen BM, Al Naiemi N, Thijsen S, Vlaminckx BJ, Fluit AC, Leverstein-van Hall MA (2010) Evaluation of Etest to determine tigecycline MICs for Enterobacter species. Antimicrob Agents Chemother 54: 2746-2747.

7. Boucher HW, Talbot GH, Bradley JS, Edwards JE, Gilbert D, Rice LB, Scheld M, Spellberg B, Bartlett J (2009) Bad bugs, no drugs: no ESKAPE! An update from the Infectious Diseases Society of America. Clin Infect Dis 48: 1-12.

8. Hope R, Pllana T, James D, Warner M, Livermore DM (2010) Zone breakpoints, by the CLSI disc method, for 15 $\mu \mathrm{g}$ tigecycline discs corresponding to EUCAST MIC breakpoints. J Antimicrob Chemother 65: 2262-2264.

9. Lu CL, Liu CY, Huang YT, Liao CH, Teng LJ, Turnidge JD, Hsueh PR (2011) Antimicrobial susceptibilities of commonly encountered bacterial isolates to fosfomycin determined by agar dilution and disk diffusion methods. Antimicrob Agents Chemother 55: 4295-4301.

10. Clinical and Laboratory Standards Institute (2009) Methods for dilution antimicrobial susceptibility tests for bacteria that grow aerobically; approved standard, 8th ed. CLSI document M7-A8 CLSI, Wayne, PA, USA.

11. Clinical Laboratory Standards Institute (2009) Performance standards for antimicrobial disc susceptibility tests; approved standard, 10th ed. CLSI document M2-A10. CLSI, Wayne, PA, USA.

12. Hope R, Mushtaq S, James D Pllana T, Warner M, Livermore DM (2010) Tigecycline activity: low resistance rates but problematic disc breakpoints revealed by a multicentre sentinel survey in the UK. J Antimicrob Chemother 65: 2602-2609. 
13. Torrico M, González N, Giménez MJ, Alou L, Sevillano D, Navarro D, Díaz-Antolín MP, Larrosa N, Aguilar L, GarciaEscribano N (2010) Influence of Media and Testing Methodology on susceptibility to tigecycline of Enterobacteriaceae with reported high tigecycline MIC. J Clin Microbiol 48: 2243-2246.

\section{Corresponding author}

Fernando G. Pasteran

Servicio Antimicrobianos

Instituto Nacional de Enfermedades Infecciosas (INEI) - ANLIS

"Dr. Carlos G. Malbrán"

Velez Sarsfield 563 Avenue (C1282AFF)

Buenos Aires, Argentina.

Telephone/FAX: 54-11-4303-2812

Email: fpasteran@anlis.gov.ar, fpasteran@gmail.com

Conflict of interests: No conflict of interests is declared. 